\title{
Study of Deflection Measurement For Bridge Using Laser Image Technology
}

\author{
Yujing Guo ${ }^{1, a}$, Li Wei ${ }^{2, b}$ \\ ${ }^{1}$ Department of Mechanical \& Electrical Enginerring, Tangshan College, Hebei Tangshan, 063000, \\ China \\ ${ }^{2}$ Department of Mechanical \& Electrical Engineering, Tangshan College, Hebei Tangshan, 063000, \\ China \\ ablueair131@163.com, bysuweili@163.com
}

Keywords: Deflection measurement; Bridge; Laser image; Wireless transmission

\begin{abstract}
Deflection is an important indicator of bridge health. This paper puts forward a deflection measurement system for bridge based on laser image technology. In the measurement progress, the laser image is sampled, filtered and converted to binary image using threshold segmentation, which is used to coordinate the center of light spot. The displacement of the location of light spot center relative to the reference position is used to calculate the deflection of bridge. The system has the features of high accuracy, low cost, and is suitable for continuous monitoring.
\end{abstract}

\section{Introduction}

Bridge is an important part of the transportation infrastructure. In order to detect the performance of bridge, it usually needs to determine the deformation of key parts of it. The deformation is caused by changes of natural conditions and long-term load even overload. It maybe bring damage when exceeds the certain threshold. So bridge health measurement must be taken in order to ensure the safe use of it. As an important parameter of bridge structure, deflection represents the deformation of bridge, so it can point out that whether the deformation of bridge is beyond the security range.

There are a lot of methods to measure the deflection of bridge. The direct measurement method of the displacement sensor and the indirect measurement method of strain measurement are used to measure the deformation. In recent years, there are some new methods of deformation monitoring with the development of computer technology, sensor technology and network technology, such as Stretched wire ${ }^{[1]}$ 、 tilt-meter ${ }^{[2]} 、$ GPS $^{[3]}$, optical fiber ${ }^{[4]}$, laser image ${ }^{[5]}$. These methods can realize real-time、 on-line and automatic measurement of bridge deflection. Moreover, the method of laser image has the advantages of convenient installation, low maintenance cost and high precision, which becomes research hotspot.

Considering the actual situation of bridge deflection measurement, this paper puts forward a bridge deflection measurement system based on laser image technology. This system can not only realize measurement at night which can not done in other methods, but also achieve high accuracy with higher price ratio.

\section{System structure}

The deflection measurement system is composed of laser emitter, laser receiver and upper computer, as shown in Figure 1. The laser beam should be long-term stability, anti-interference and high positioning accuracy, in order to ensure the accurate positioning of the spot center. A diode pump solid state laser(DPSSL) with reliable stability and limberness of light spot shape is used in the study. 


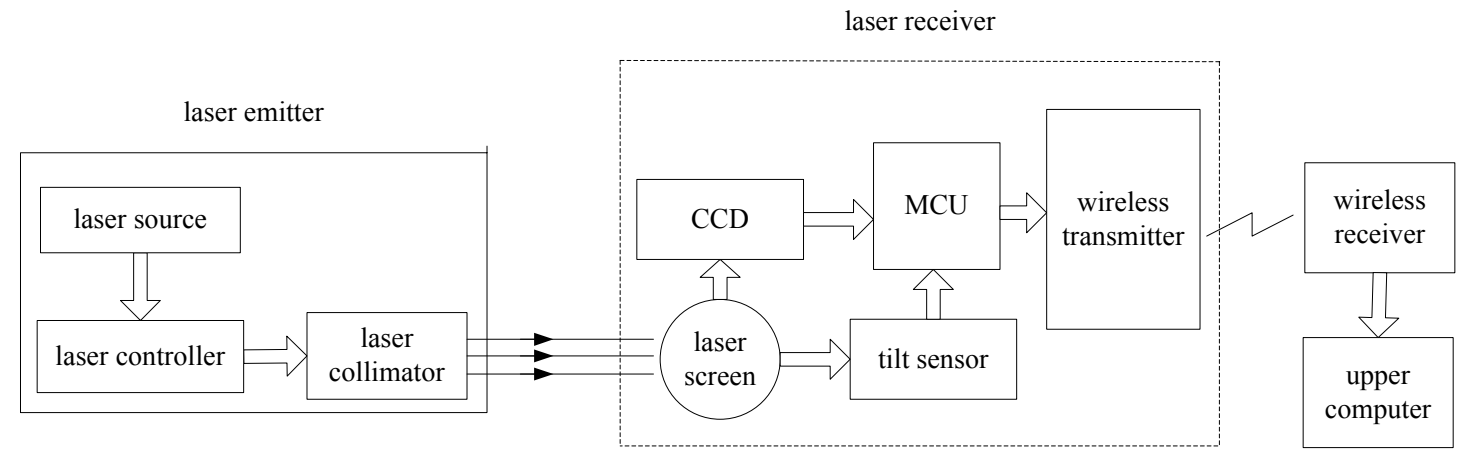

Fig.1. The structure of bridge deflection measurement system

\section{Basic principles}

Laser emitter is installed on the measurement base point and laser receiver is fixed at the measurement point of bridge. Laser projects on the fixed laser screen. Laser image will be sampled by CCD and sent to the MCU. A laser spot is formed in the imaging, and it is processed by digital image processing technology. The coordinate of the center of the laser spot will be calculated. With deformation of the bridge, the position of the center of the laser spot is changed. This variation is equal to the deflection of bridge, so the deflection of bridge can be obtained by calculating the variation of the center of the laser spot.

When the bridge deformation occurs, laser receiver will also have a slight angle of inclination,as shown in Figure 2. In order to reduce measurement error, this study introduces tilt sensor to measure the angle of the laser emitter.

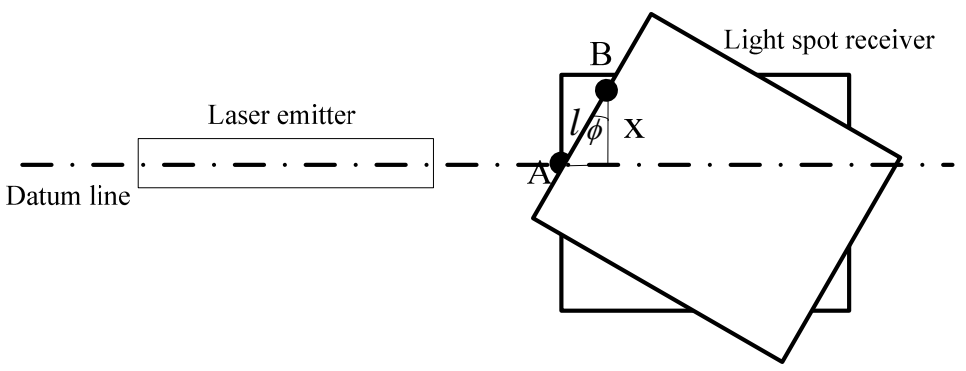

Fig.2. Principle figure of deformation measurement

As shown in the Figure 2, it designates that $\mathrm{A}$ is the datum position, $\mathrm{B}$ is the laser light spot position, $l$ is the length of $\mathrm{AB}$ line, which is the deformation relative to datum plane. $\phi$ is the obliquity of light sot receiver relative to datum plane. $\Delta$ is the displacement value of the measured point perpendicular to the datum plane, so $\Delta$ is the deformation result. The relationship between the three is shown in the eq.(1). The vertical deformation of the position can be analyzed by collecting the center coordinates of the spot image.

$$
\Delta=l \cos \phi
$$

\section{Laboratory study}

Laser emitter is composed of laser power, laser controller and laser collimator; Laser receiver is composed of laser screen, CCD, tilt sensor, MCU and wireless transmitter.Laser emitter and Laser receiver are shown in Figure 3 and Figure 4. 


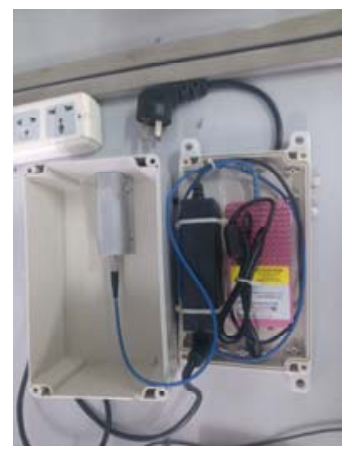

Fig.3. Laser emitter

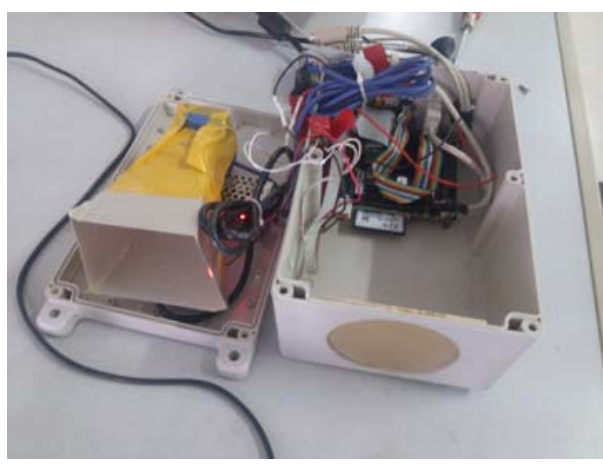

Fig.4. Laser receiver

Upper computer module is composed of wireless receiver and computer.

Usually, the measuring device was installed on the bridge, and the upper computer as the monitor is far from the measured point, so this system uses the wireless way to transmit the signal.

The laser image on CCD has not only a light spot, but also fringes due to diffraction and scattered light, as shown in Figure 5. The reason for this situation is that: 1. The distance between laser emitter and laser receiver is long; 2 .The natural light in measurement environment causes certain interference to laser imaging.

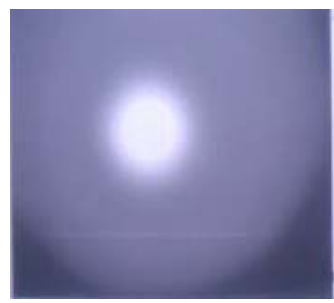

Fig.5. Laser image

The brightness of fringes overall is much lower than the brightness of the light spot. Therefore, the image can be processed by filtering , threshold segmentation to remove the influence of diffraction light s scattered light and natural light, and converted to a binary image. The laser image after processing is shown in Figure 6.

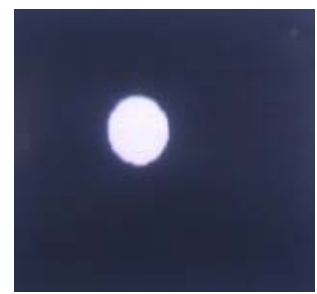

Fig.6. Laser image after filtering and threshold segmentation

In Figure 6, the edge of the light spot is smooth and clear, and basically unchanged compared with Figure 5. So the coordinate of the center of the light spot in Figure 6 can be accurately computed.

The signals of laser image and angle of inclination were sampled by MCU in laser receiver. Signal processing and result display are done by the upper computer. The upper computer display interface is shown in Figure 7.

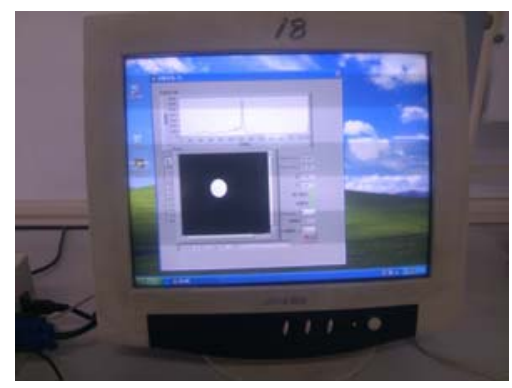

Fig.7. Display interface of deflection measurement system 
According to the requirement of the measurement environment, the angle sensor LCA320T with small size, low power consumption and dual axis voltage output is selected. The sensor uses the non-contact measurement principle. The angle and the output voltage is linear and the output voltage is transmitted to the computer through two channels. As shown in Figure 7, the front panel shows the pattern of the measurement module, the gray histogram and the center coordinates and the dip angle of the spot.

\section{Conclusions}

This paper puts forward a system for the detection of bridge deflection. It makes use of the characteristics of high precision of laser displacement sensor 、 low dependence on the light in measurement environment. It can reduce measurement error through image processing technology. The system realizes the measurement of bridge deflection, and showes a good application future.

\section{References}

[1] Lan Zhangli, Yang Xiaofan: Non-contact Bridge Deflection Measurement System Using Weighted-stretched-wire. Chinese Journal of Scientific Instrument. 2008 (29)1058-1062

[2] He Yongqi, Chen Weimin , Fu Yumei: Bridge Deformation Monitoring with Tilt Meter. Journal of Chongqing University. 2004 (27)28-31

[3] Li Chuanjun, Wang Qing, Liu Yuanqing, etc: The Application of Deflection Deformation Survey with GPS for the Suspended Bridge of Runyang Large Bridge. Geotechnical Investigation \& Surveying.2010 (3)65-68

[4] Zhang Kuangwei, Zhang Shaojie, Zhao Xiaoxia, etc: Application of FBG Strain Sensors in Bridge in Bridge Structure Monitoring System. 2014 (36)15-19

[5] Zhang Benniu, Li Xingxing, Song Jun: Application Study on Laser Projection Sensor for Bridge Deflection Detection. Chinese Journal of Sensors and Actuators, 2009 (22)755-759 